\title{
Examination of Graphene in a Scanning Low Energy Electron Microscope.
}

\author{
Ilona Müllerová, Eliška Mikmeková and Luděk Frank
}

Department of Electron Microscopy, Institute of Scientific Instruments ASCR, v.v.i., Brno, Czech Republic.

Although graphene has been available [1-2] and intensively studied [3-5] for a full decade, new methods are still required for its examination and diagnostics. Even checking the continuity of layers and the reliable counting of layers of graphene and other 2D crystals should be easier to perform. Scanning low energy electron microscope (SLEEM) equipped with a cathode lens [6] offers an innovative tool enabling one to see graphene samples at nanometer lateral resolution in both transmitted and reflected electrons and to count the number of layers. This diagnostics can be performed on freestanding graphene samples as well as on graphene grown on the surfaces of bulk substrates.

The freestanding graphene samples were first examined in the standard vacuum high resolution SLEEM. Fig. 1 shows micrographs taken in the reflected electron (RE) as well as transmitted electron (TE) mode at several energies. The RE signal was composed of both secondary and backscattered electron emission, accelerated in the cathode lens field toward the detector. In the RE frames the maximum contrast between the graphene layers and lacey carbon appears at $1 \mathrm{keV}$ and decreases toward higher and lower energies because of extending and shortening information depth, respectively. These images identify empty holes but do not reveal thicker islands of graphene. In the TE mode we do not see multilayer graphene islands above $100 \mathrm{eV}$. This fact underlines the suitability of very low energy electron microscopy for examination of 2D crystals. Interpretation challenges are presented by some details inverting their contrast more than once, see the arrow. These probably arise from contaminations that become charged.

Usually used counting of graphene layers by Raman spectroscopy is faced by the issue of the low lateral resolution of light optical imaging. SLEEM provides much higher resolution, so it is worth checking its selectivity for the same purpose. Fig. 2 left shows that the contrast of individual graphene layers is preserved down to units of $\mathrm{eV}$. Measurement of the transmissivity was calibrated between the zero signals on mesh rungs and the full signal in empty holes, see Fig. 2 and Table 1.

The transmissivity of the graphene samples naturally depends on cleanness of the surface. We have established that while fast electrons decompose the adsorbed hydrocarbon molecules creating a carbonaceous contamination layer, below $50 \mathrm{eV}$ electrons release these molecules and leave surface of the graphene atomically clean, see Fig. 3 [7].

\section{References:}

[1] KS Novoselov et al, Science 306 (2004) p. 666.

[3] JC Meyer et al, Nano Letters 8 (2008) p. 3582.

[4] PY Huang et al, Nature 469 (2011) p. 389.

[5] W Zhou et al, Microscopy and Microanalysis 18 (2012) p. 1342.

[6] I Müllerová and L Frank, Advances in Imaging and Electron Physics 128 (2003) p. 309.

[7] The authors acknowledge funding from the Technology Agency of the Czech Republic (Competence center Electron microscopy, no: TE01020118) and from the MEYS of the Czech Republic (LO1212). 

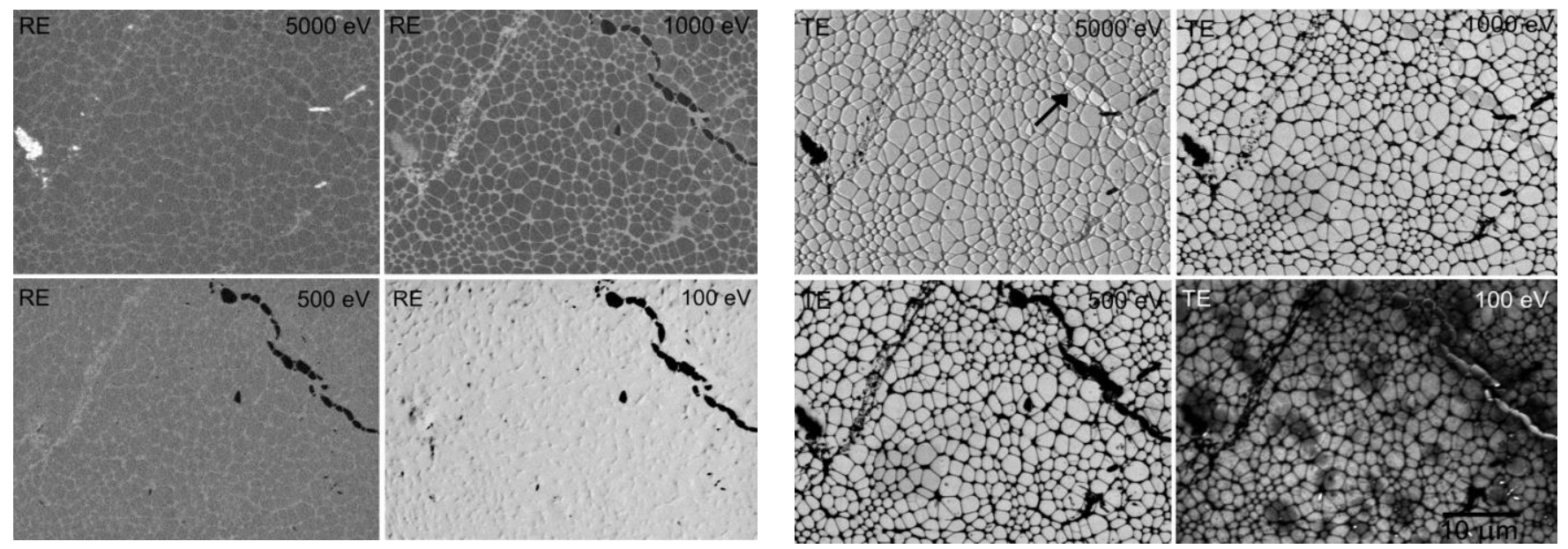

Figure 1. Freestanding graphene sample of 3 to 5 layers imaged in reflected and tramnsmitted electrons.

Table 1: Total transmissivity measured on graphene samples for $40 \mathrm{eV}$ incident electrons.

\begin{tabular}{|l|l|l|l|l|l|l|l|}
\hline No. of graphene layers & 1 & 2 & 3 & 4 & 5 & 6 & 7 \\
\hline Transmissivity (\%) & 11.9 & 9.0 & 6.5 & 4.9 & 3.5 & 2.6 & 2.0 \\
\hline
\end{tabular}
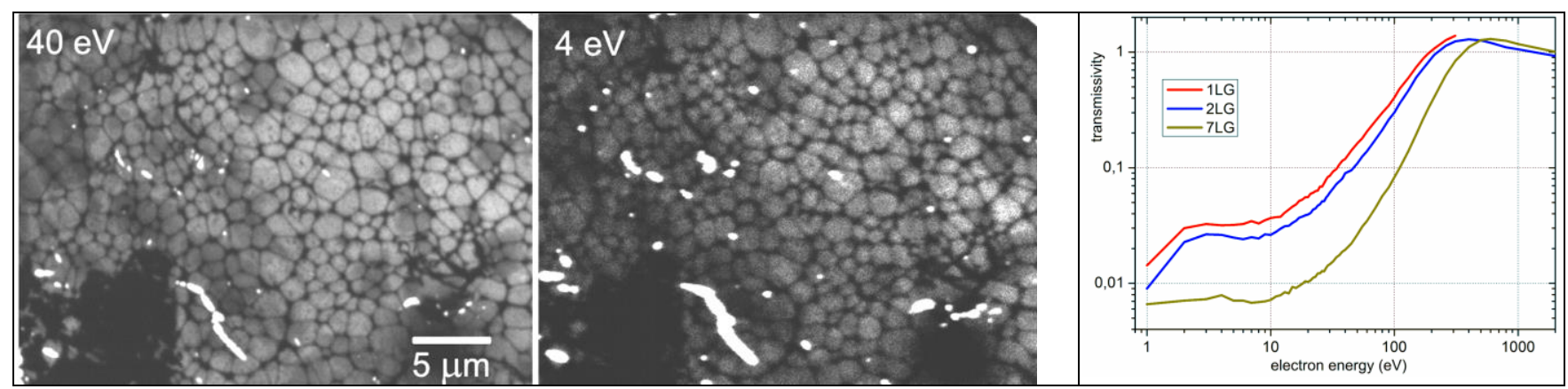

Figure 2. Micrographs of a 3to5LG graphene sample taken in a UHV microscope (left). The measured energy dependence of transmissivity (right).
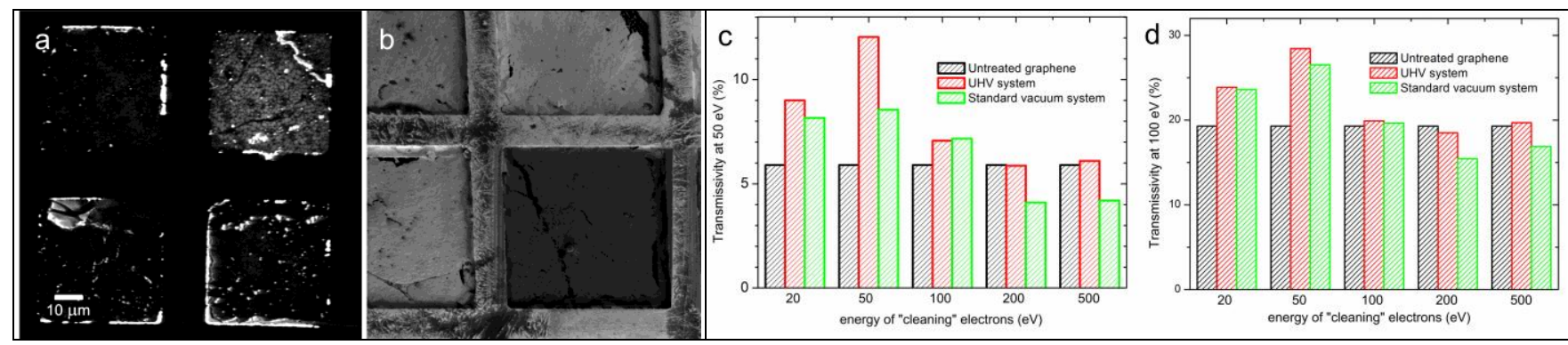

Figure 3. Changes in properties of $1 \mathrm{LG}$ due to prolonged bombardment with $30 \mathrm{eV}$ electrons shown in the transmission (a) and reflection (b) modes. Quantitative development of 1LG transmissivity at $50 \mathrm{eV}$ (c) and $100 \mathrm{eV}$ (d) in dependence on vacuum conditions and the energy of electrons used for prolonged bombardment. 